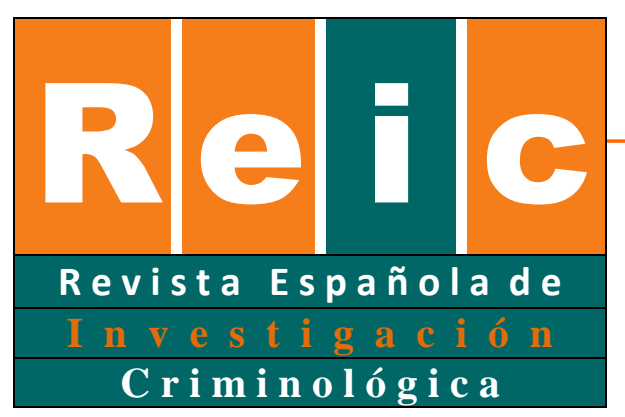

\title{
Mujeres a la sombra: Influencia del género en las actitudes profesionales de los funcionarios penitenciarios ${ }^{1}$
}

Manuscrito recibido el 23 de diciembre 2012 / Publicado el 27 mayo 2013

\author{
David Cuaresma \& Laura Nicolás \\ Universitat de Barcelona
}

\begin{abstract}
RESUMEN
En este trabajo se estudian las diferencias entre géneros en las actitudes mostradas por aspirantes al cuerpo de funcionarios de prisiones en relación con siete aspectos ligados a sus funciones profesionales como por ejemplo: apoyo a la rehabilitación, autopercepción profesional como trabajadores o la que manifiestan respecto a los presos sobre quienes tienen atribuida la responsabilidad de custodia y vigilancia. Se estudia también cómo la variable género puede condicionar el rendimiento profesional de los funcionarios responsables de la seguridad penitenciaria. Este análisis se llevó a cabo contrastando las actitudes mostradas antes y después del período de formación y capacitación inicial, comprobando también el efecto de la formación profesional recibida. Se constata el efecto de la variable género. ${ }^{2}$
\end{abstract}

\footnotetext{
${ }^{1}$ La correspondencia debe enviarse a D. Cuaresma. Facultat de Psicologia. Universitat de Barcelona. Pg. de la Vall d'Hebron, 171, 08035 Barcelona (Spain). dcuaresma@ub.edu

2 Se sigue aquí el criterio establecido por la OMS (http://www.who.int/gender/whatisgender/en/) al distinguir entre sexo y género, atribuyendo al primer término las características biológicas y fisiológicas que definen a hombres y mujeres, mientras que reserva para el segundo la referencia a los roles socialmente construidos, comportamientos, actividades y atributos que una sociedad considera apropiados para hombres y mujeres.
} 
Palabras clave: funcionarios de prisiones, actitudes profesionales, política penitenciaria, diferencias de género, sistema penitenciario, formación y competencias profesionales, motivación, autoeficacia

\begin{abstract}
In this paper we study the gender differences in attitudes displayed by candidates applying for the role of prison officers, regarding seven aspects of their professional duties, such as rehabilitation-support, self-perception as workers, or attitudes towards the prisoners with whose custody and oversight they are charged. Moreover we study how the gender variable can have a bearing on the job performance of prison officers. This analysis was conducted contrasting attitudes held before and after the initial training, testing the effect of professional training received. The effect of the gender variable is evidenced.
\end{abstract}

Key words: prison officers, professional attitudes, prison policy, gender differences, penitentiary system, training and skills, motivation, self-efficacy

\title{
1. Introducción
}

Tradicionalmente los estudios sociales sobre profesiones han destacado en los ámbitos relacionados con el mundo del derecho o la medicina, ya que estas eran consideradas profesiones clásicas con un prestigio reconocido ante la ciudadanía. Estas profesiones cuentan con un amplio bagaje de conocimientos y estudios previos sobre su composición, funciones, carrera profesional, formación y todos aquellos aspectos que sirven para identificarlos como ámbitos significativamente diferenciados y marcadamente peculiares dentro del desempeño de los oficios.

No obstante no pocos criminólogos y también algunos sociólogos se han interesado por el estudio de las profesiones relacionadas con la seguridad pública, conformando un núcleo de conocimiento suficientemente amplio como para poder hablar ya de la existencia de una tradición de estudio en el caso concreto de la profesión policial que ha concitado el interés de numerosos autores a lo largo de la segunda mitad del siglo XX (Ballbé, 1983; Jar, 1995; López, 1987; Marín, 1990; Torrente, 1997).

Aun así el trabajo de los funcionarios de prisiones no parece haber tenido hasta ahora el mismo atractivo científico, un interés que sí han provocado las instituciones carcelarias en su conjunto y, muy especialmente, los internos en particular. De este modo no es muy frecuente encontrar en la literatura científica de habla hispana estudios sobre el ejercicio profesional penitenciario, pese a las excepciones que pueden 
representar los trabajos de De Diego et al. (1997), Durán (2007), Giménez-Salinas (1990), Luque et al. (1992) o Sarasa (1990). En cambio, en la literatura internacional sí es más frecuente encontrar referencias sobre este particular aspecto del funcionamiento del sistema penal. Pueden servir como ejemplo de ello los trabajos de Bensimon (2004; 2005a; 2005b), Crouch y Alpert (1982), Kauffman (1988), Lambert et al. (2010), Liebling y Price (2001), Malochet (2004), Tellier y Serin (2001) o Williamson (1990), destacando, por su calidad, el estudio de Crawley (2004). Quizá esta falta de atención en España, y también en Hispanoamérica, sea debida a que los funcionarios penitenciarios, $\mathrm{y}$ en particular los responsables de la seguridad, han sido vistos como un elemento negativo e incluso indeseable aunque necesario del sistema carcelario (Cuaresma y Nicolás, 2006; Garriga, 2000).

Así pues muy poco sabemos sobre las actitudes profesionales de estos hombres y mujeres, y aún menos de las interacciones que mantienen con los internos y de cómo unas y otras pueden afectar al éxito de su misión, pese a que son quienes en mayor medida mantienen contacto directo con los internos ya que tienen entre sus funciones principales la seguridad pública: a través de la reinserción de los reclusos y del aseguramiento del mandato de reclusión al que éstos han sido sometidos, ofreciéndoles a su vez asesoramiento y apoyo al tiempo que garantizan la seguridad interior de las prisiones.

Algunos de los trabajos mencionados han descrito una subcultura profesional propia del medio penitenciario que, tomando elementos de la subcultura delictivacarcelaria y de los ambientes marginales, se ha conformado como seña de identidad de la profesión (Crawley, 2004). No se trata de un caso aislado ni especialmente singular, otras profesiones elaboran sus propios códigos de conducta, creencias, actitudes, ritos, costumbres e incluso jergas. Los procesos de socialización organizacional se caracterizan porque intentan adherir al nuevo sujeto a la organización. Para ello el nuevo miembro tiene que aprender la escala de valores, las normas y las pautas de conducta que exige cada organización (Goffman, 1970). En el caso de los funcionarios penitenciarios esta realidad está quizá más acentuada debido al carácter cerrado y muchas veces opresivo del contexto carcelario, con una constante percepción de peligro o amenaza, y con una carga emocional muy acentuada. Todos estos rasgos acostumbran a describirse como presentes en el día a día del trabajo en prisión (Bensimon, 2004; 
Crawley, 2004; Durán, 2007; Liebling y Price (2001); Lavoie et al., 2006). Estas características subculturales conforman una serie de respuestas emocionales, cognitivas y comportamentales que son en algunos aspectos muy similares a las que se han encontrado en el trabajo policial (tendencia al cinismo, sospecha, machismo y un fuerte sentimiento de solidaridad en el grupo), características que están presentes en todos los entornos penitenciarios, pese a que su intensidad depende de ciertas condiciones del entorno laboral y del contexto en el que este se desarrolle (Crawley, 2004).

En este ambiente aparentemente muy masculinizado las mujeres parecen tener un difícil acomodo, y a pesar de ello el sistema penitenciario catalán no parece caracterizarse por ser precisamente resistente a la incorporación de la mujer.

En las cuatro últimas convocatorias públicas de oposición al cuerpo de funcionarios de prisiones en Cataluña la proporción de mujeres se situó entre el 40,7\% y el 49,7\%. Esto ha situado en marzo de 2013 el porcentaje de mujeres en este cuerpo en un $36,1 \%$. Esto contrasta con el conjunto de la administración pública catalana, donde el colectivo femenino representa un $65,5 \%$ del total ${ }^{3}$. No obstante esta proporción es muy inferior en las profesiones relacionadas con la seguridad pública, donde difícilmente se alcanzan porcentajes de presencia femenina superiores al 25\%. Así por ejemplo en la misma fecha, marzo de 2013, en el cuerpo de Policia de la Generalitat-Mossos d'Esquadra las mujeres representan el $21,4 \%$ y el $11,6 \%$ en el de Agents rurals ${ }^{4}$.

Si se toma en consideración la edad de los funcionarios aún se aprecia mejor el incremento femenino: así el 80\% de las funcionarias en ejercicio al finalizar el año 2008 tenían una edad comprendida entre los 18 y los 45 años, mientras que los hombres en esa misma franja de edad representaban el $68 \%$ de su grupo. Asimismo los funcionarios penitenciarios entre 18 y 30 años son en un $42 \%$ mujeres, y el hecho de que en las últimas promociones la representación de géneros se acercase a la paridad, tal y como ya se ha visto, permite afirmar que hay en marcha un proceso de feminización del colectivo. Este fenómeno es especialmente relevante si tenemos en cuenta que, como es bien conocido, en torno al $93 \%$ de los internos penitenciarios son hombres.

\footnotetext{
${ }^{3}$ Con estos datos la administración pública catalana se sitúa muy por encima de la paridad que el Consejo de Europa promueve mediante sus políticas de igualdad, en particular la Recomendación 96/694, de 2 de diciembre de 1996, DO L 319.

4 Según datos de la Generalitat de Catalunya. Obtenidos el 12 de abril de 2013 de la página http://analisiocupaciopublica.gencat.cat/
} 
El proceso de feminización del cuerpo de seguridad penitenciaria no es en realidad nuevo, la alta presencia de mujeres ha sido constante a lo largo de los últimos años en el sistema penitenciario catalán, así por ejemplo en 1991 el 38\% de los nuevos funcionarios fueron mujeres (Luque et al., 1992). Este fenómeno se repite en otros sistemas penitenciarios: en el caso de Canadá, con un 47,2\% de mujeres en los cursos selectivos del año 2005 (Bensimon, 2005a), y algo similar se ha producido también en el caso francés desde el año 2000 (Malochet, 2005). En EE.UU. cerca de un tercio del personal penitenciario dedicado a la seguridad está integrado por mujeres (Lambert et al. 2010), parecido porcentaje al de Nueva Zelanda (Newbold, 2005). Otros en cambio mantienen niveles sustancialmente inferiores, por ejemplo en el sistema penitenciario taiwanés las funcionarias suponen el 20\% (Lai et al. 2012).

Las actitudes profesionales evidenciadas por los aspirantes al ámbito penitenciario de seguridad, en el contexto de la formación inicial selectiva previa, y las diferencias actitudinales entre hombres y mujeres fueron el objeto de este trabajo.

\section{Marco teórico}

Por lo que respecta al rol profesional y a las funciones que desempeñan los funcionarios penitenciarios un reciente estudio realizado con funcionarios de prisiones norteamericanos lo resumió en los siguientes términos: "Su rol implica ayudar a los internos a hacer frente a su encarcelamiento, asistirles con los problemas institucionales y dirigir a los internos angustiados a las fuentes adecuadas (...). Los funcionarios de prisiones son responsables de crear y mantener un ambiente humano tras los muros. Asumen un rol directo en la solución de los problemas de los internos, controlando las disputas entre ellos, disciplinándolos y actuando como intermediarios entre los internos y el resto de estamentos de la burocracia penitenciaria” (Farkas, 1999; p. 496). Por su parte para Bensimon (2005a; p. 56) los funcionarios de prisiones "son agentes de policía 'interior', el trabajo de los cuales no es encontrar y detener personas que hayan cometido delitos, sino mantener a esas personas (...) en un área restringida”, lo que la Ley Orgánica General Penitenciaria (LOGP) viene a denominar "retención y custodia". Abundando en la similitud entre trabajo policial y penitenciario numerosas sentencias 
han puesto de relieve sus semejanzas, asentando jurídicamente la condición de agente de la autoridad de los funcionarios responsables de la seguridad interior de los establecimientos penitenciarios.

Para poder llevar a cabo un estudio como el presente es necesario acotar antes no solo el rol profesional que desarrollan los funcionarios, sino también definir un modelo de competencias profesionales. Pero llevar a cabo esta labor previa no es tarea sencilla. En este trabajo se tuvo en cuenta el modelo de competencias profesionales desarrollado por el Centre d'Estudis Jurídics i Formació Especialitzada (CEJFE) y el Departament de Justícia de la Generalitat de Catalunya, así como el propuesto por Cuaresma y Nicolás (2006).

Como se verá el modelo de competencias sirvió para definir las variables analizadas e incluía los siguientes diez ítems:

\section{Tabla 1: Modelo de competencias profesionales de los funcionarios penitenciarios} en Cataluña

\begin{tabular}{|l|l|}
\hline 1. Control para la seguridad & $\begin{array}{l}\text { Reconocimiento de la necesidad de control y } \\
\text { habilidad para el mantenimiento del mismo sobre } \\
\text { métodos, personas y materiales. }\end{array}$ \\
\hline 2. Prevención de incidencias & $\begin{array}{l}\text { Habilidad para llevar a cabo un análisis lógico y } \\
\text { rápido de los indicios observados identificar } \\
\text { problemas, recoger información significativa y } \\
\text { relacionar datos relevantes. }\end{array}$ \\
\hline 3. Gestión de conflictos & $\begin{array}{l}\text { Habilidad para intervenir en situaciones de riesgo } \\
\text { respondiendo con argumentos sólidos, persuadir y } \\
\text { hacer que los otros cambien de conducta o actitud a } \\
\text { través de una interacción social o del uso de medidas } \\
\text { disciplinarias o de la fuerza. }\end{array}$ \\
\hline 4. Comunicación con los & $\begin{array}{l}\text { Habilidad para exponer información de forma clara y } \\
\text { internos }\end{array}$ \\
\hline
\end{tabular}




\begin{tabular}{|c|c|}
\hline $\begin{array}{l}\text { 5. Cooperación y trabajo en } \\
\text { equipo }\end{array}$ & $\begin{array}{l}\text { Habilidad para desarrollar y mantener relaciones } \\
\text { laborales positivas y disposición para aceptar } \\
\text { responsabilidades, resolver conflictos y participar } \\
\text { activamente en la obtención de una meta común con } \\
\text { el resto de personal penitenciario. }\end{array}$ \\
\hline 6. Inici & $\begin{array}{l}\text { Habilidad para resolver de forma eficaz las } \\
\text { situaciones que surgen en el día a día de la actividad } \\
\text { sin efectuar todas las consultas a la línea jerárquica, } \\
\text { evitando así la agravación de problemas de } \\
\text { importancia menor. }\end{array}$ \\
\hline $\begin{array}{l}\text { 7. Adaptabilidad y } \\
\text { flexibilidad }\end{array}$ & $\begin{array}{l}\text { Habilidad para adaptarse a las normas y al entorno } \\
\text { penitenciario. Habilidad para adaptar el propio } \\
\text { comportamiento a diferentes contextos, situaciones, } \\
\text { medios y personas de forma rápida y adecuada sin } \\
\text { perder en ningún momento el sentido del rol. }\end{array}$ \\
\hline 8. Tolerancia a la $\mathrm{p}$ & $\begin{array}{l}\text { Habilidad para seguir trabajando con eficacia bajo la } \\
\text { presión ambiental o el estrés. }\end{array}$ \\
\hline 9. Organización del trabajo & $\begin{array}{l}\text { Habilidad para organizar las tareas del servicio al } \\
\text { cual ha sido asignado. }\end{array}$ \\
\hline 10. Gestión de la información & $\begin{array}{l}\text { Habilidad para observar el entorno, recoger } \\
\text { información relevante y transmitirla al resto de } \\
\text { personal de forma adecuada. }\end{array}$ \\
\hline
\end{tabular}

El éxito o el fracaso de las instituciones penitenciarias depende del personal que trabaja en ellas (Paoline et al., 2006; Redondo, 2008), en consecuencia resulta conveniente que este personal, en especial el personal de vigilancia, quien mantiene un contacto más intensivo y permanente con las personas encarceladas, y el sueldo de los cuales representa normalmente el primer gasto de cualquier administración penitenciaria, reciba una formación especializada y de calidad que les permita afrontar los grandes retos que plantea un penitenciarismo democrático, respetuoso con la dignidad y los derechos humanos, y enfocado a la reintegración en la sociedad antes que a la pura retribución o castigo del transgresor penal. Así lo afirma el Consejo de Europa, 
en el principio fundamental número 8 de las Normas penitenciarias europeas ${ }^{5}$, cuando dice que "El personal penitenciario lleva a cabo una importante misión de servicio público y su reclutamiento, formación y condiciones de trabajo le deben permitir proveerse de un alto nivel al hacerse cargo de las prisiones", también el artículo 81 de esta norma dedicado en exclusiva a la formación del personal penitenciario abunda en esa necesidad. Atendiendo a la complejidad de las tareas y la evolución del sistema penitenciario, y de las profesiones que en su seno se desarrollan, todo parece indicar que los oficios penitenciarios no pueden ser ejercidos sin el apoyo de una formación sólida. No en vano se ha afirmado que la selección, la valoración y la formación del personal de prisiones van ligadas a las habilidades actitudinales y de comportamiento específicas que se requieren para el éxito de la misión y el trabajo penitenciario (Bensimon, 2004, 2005a, 2005b; Motiuk y Serin, 2001). Por otra parte la formación de los funcionarios es un proceso de enculturación que puede resultar lento, desafiante y complejo (Crawley, 2004), pero que representa un elemento dinamizador del cambio que puede contribuir a incrementar y mejorar el rendimiento institucional de los centros penitenciarios (Bensimon, 2004; Cuaresma y Nicolas, 2006; Ramió, 2006).

Por otra parte, en relación con el proceso de feminización apuntado más arriba y en un sentido general, trascendiendo la perspectiva meramente profesional y siguiendo en ello a Pinker (2011), se puede afirmar que la feminización produce en sí misma un efecto beneficioso, ya que las sociedades en que las mujeres ostentan más poder tienden a alejarse de la glorificación de la violencia y son menos propensas a desarrollar en su seno peligrosas subculturas de jóvenes desarraigados, optando en mayor medida por resolver los conflictos mediante la negociación y el acuerdo antes que mediante la violencia

En particular en lo relativo al ámbito profesional penitenciario, y en base a la revisión de la investigación internacional, se plantea que la variable género debe tener necesariamente su reflejo en las relaciones que se establecen a nivel laboral y personal entre los funcionarios, y entre éstos y los internos de ambos géneros. Una prueba de estas relaciones profesionales particulares la encontramos en la siguiente afirmación de Crawley (2004; p. 250-251): "Los funcionarios tienen que ser capaces de demostrar

\footnotetext{
${ }^{5}$ Recomendación Rec (2006) 2 del Comité de Ministros de los Estados miembros sobre las Reglas Penitenciarias Europeas, adoptado por el Comité de Ministros el 11 de enero de 2006 en la 952a Reunión de Delegados de Ministros.
} 
alguna versión de la imagen 'masculina' del funcionario penitenciario; para las funcionarias, en cambio (percibidas por muchos funcionarios masculinos como influencia 'calmante' o 'civilizadora' de los presos) una imagen de 'macho' es innecesaria (aunque como hemos visto, algunas funcionarias escojan mostrarla)". Esta misma autora nos informa que la alta representación femenina suele ser considerada fuente de conflictividad laboral, siendo a juicio de sus compañeros incluso percibidas como pasivas, indefensas y manipuladoras. Asimismo se aduce con frecuencia la incompatibilidad de su condición sexual con el cumplimento de determinadas funciones propias de las profesiones relacionadas con la seguridad, poniéndose como recurrente ejemplo de ello la imposibilidad legal de que algunas funciones como los registros personales o cacheos sean realizados por profesionales de diferente sexo al de los presos (Farnworth, 1992; Griffin, 2012; Newbold, 2005) o incluso una mayor susceptibilidad a implicarse en relaciones sentimentales con los internos (Newbold, 2005). También Malochet (2005) aprecia notables diferencias en las relaciones que se establecen entre los internos y los funcionarios de uno y otro sexo. Según este autor los internos de género masculino parecen mostrar hacia las funcionarias un respeto debido a su condición de mujer más que a la autoridad de su cargo; mientras que las funcionarias, ante situaciones de agresividad o violencia protagonizadas por los internos, adoptan hacia ellos una actitud más dialogante, propensa a la negociación y a la diplomacia en lugar de preferir recurrir a la disuasión o incluso al uso de la fuerza. Por su parte Taxman y Gordon (2009) y Gordon et al. (2012) apreciaron una mayor propensión de las funcionarias a experimentar miedo de ser víctimas de actos delictivos en el ejercicio de su trabajo. Finalmente, centrando la cuestión en el ámbito formativo de reclutamiento inicial, las vivencias de las funcionarias resumen este proceso con términos como “abuso verbal, ridículo y humillación ritual”, y lo identifican como "el castigo por entrar en una profesión dominada por los hombres" (Crawley, 2004; p. 69).

Las conclusiones de la investigación internacional no son concluyentes al respecto de las posibles diferencias entre los dos géneros por lo que respecta al ejercicio profesional como funcionario de prisiones. Así por ejemplo según Farkas (1999) el género fue identificado como una variable relevante y que se relacionaba significativamente con la orientación a la rehabilitación. Las funcionarias mostraban una mayor preferencia por ejercer roles de apoyo y asesoramiento y eran punitivas en 
sus actitudes hacia el delito. También Morgan et al. (2002) encontraron diferencias, apreciando en las funcionarias una menor tendencia al trato impersonal con los internos, como consecuencia del efecto del síndrome de burnout. Estos autores sugieren que hay una mayor capacidad femenina para ajustarse a los estresores profesionales de la prisión. Por su parte Ireland y Quinn (2007) no encontraron relación entre el género y las actitudes hacia los internos aunque sí apuntaron la necesidad de fomentar la empatía en los aspirantes de género masculino durante el periodo de formación. Estos autores abogan asimismo por fomentar las actitudes positivas durante el periodo de formación como medio para reducir las atribuciones negativas y los prejuicios de los funcionarios hacia los internos, especialmente en el caso de los funcionarios de género masculino. Más recientemente, Lambert et al. (2010) hallaron pequeñas diferencias en la forma en que agentes femeninos y masculinos responden a la supervisión institucional.

Así pues, en un momento de aumento de la población reclusa, de creación de nuevas infraestructuras penitenciarias y, en consecuencia, de incremento de las plantillas de seguridad ${ }^{6}$, resultó oportuno determinar las siguientes hipótesis:

(1) Las actitudes profesionales variarán en función del género de los aspirantes, de forma que hombres y mujeres evidenciarán diferentes actitudes profesionales atribuibles a la variable independiente género, y

(2) Al finalizar el curso selectivo la formación recibida habrá provocado una variación de las actitudes profesionales en los aspirantes al cuerpo de seguridad penitenciaria.

\section{Metodología}

\subsection{Grupos de análisis}

\footnotetext{
${ }^{6}$ En total entre los años 2006 y 2011 la plantilla funcionarial de seguridad penitenciaria en Cataluña se incrementó en más de 1.500 efectivos. Asimismo en el caso del cuerpo de ayudantes de instituciones penitenciarias, dependiente del Ministerio del Interior, las convocatorias correspondientes a los años 2008 y 2009 sumaron más de 2.100 nuevas plazas. No obstante esta tendencia se ha visto truncada y desde 2011, lejos de incrementarse los efectivos, la dotación de profesionales penitenciarios de seguridad en Cataluña se ha visto reducida en prácticamente 400 funcionarios. Asimismo el aumento de la población reclusa se ha estancado e incluso retraído en los últimos años.
} 
Se contó con dos grupos de aspirantes que a lo largo de los años 2007 y 2008 completaron el proceso de formación inicial selectiva mediante la superación de un curso teórico-práctico. El curso se configuró mediante una primera parte de formación teórico-práctica de 9 semanas de duración (240 horas), impartida en las instalaciones del Instituto de Seguridad Pública de Cataluña (ISPC), en Mollet del Vallés. Al inicio (Pre) y término (Post) del curso los aspirantes cumplimentaron los cuestionarios actitudinales. Quienes superaron esta primera parte se incorporaron a un período de prácticas en destinos penitenciarios por espacio de 8 semanas más (280 horas). El diseño de las materias y los contenidos del curso respondían al perfil de competencias profesionales de los técnicos especialistas de servicios penitenciarios llevado a cabo por el CEJFE de conformidad con los requerimientos de la administración penitenciaria. El equipo de docentes estaba integrado por miembros de los cuerpos funcionariales del sistema penitenciario, y en su gran mayoría pertenecían al cuerpo técnico de especialistas de servicios penitenciarios, en sus diferentes categorías.

Ambos grupos conformaron el universo total de la muestra, 636 individuos: 322 aspirantes en el curso realizado el año 2007 y 314 en el de 2008. Los dos grupos tenían un perfil socio-demográfico homogéneo. Todos habían superado las pruebas selectivas que hasta ese momento se habían realizado en el proceso de oposición. 
Tabla 2: Resumen de las características socio-demográficas de cada grupo

\begin{tabular}{|l|r|r|r|r|}
\hline & \multicolumn{2}{|c|}{$\begin{array}{c}\mathbf{2 0 0 7} \\
(\mathbf{n = 3 2 2})\end{array}$} & \multicolumn{2}{c|}{$\begin{array}{c}\mathbf{3 0 0 8} \\
(\mathbf{n}=\mathbf{3 1 4})\end{array}$} \\
\hline & \multicolumn{1}{|c|}{$\%$} & $\bar{\chi} \pm(s d)$ & $\%$ & $\bar{\chi} \pm(s d)$ \\
\hline Sexo & & & & \\
\hline Mujeres & 40,7 & & 49,7 & \\
\hline Hombres & 59,3 & & 50,3 & \\
\hline Edad & & $34( \pm 6,9)$ & & $34( \pm 6,3)$ \\
\hline De 18 a 25 años & 9,9 & & 5,7 & \\
\hline De 26 a 35 años & 53,1 & & 57,0 & \\
\hline De 36 a 45 años & 30,1 & & 32,5 & \\
\hline De 46 a 55 años & 6,8 & & 4,8 & \\
\hline Formación reglada & & & & \\
\hline Ciclos formativos de grado medio & 4,4 & & 3,2 & \\
\hline Ciclos formativos de grado superior & 15,7 & & 18,4 & \\
\hline Bachillerato & 32,0 & & 37,4 & \\
\hline Diplomados & 22,9 & & 20,6 & \\
\hline Licenciados & 25,1 & & 20,3 & \\
\hline Lugar de nacimiento & & & & \\
\hline Barcelona & 47,2 & & 52,7 & \\
\hline Girona & 1,6 & & 1,6 & \\
\hline Lleida & 31,0 & & 30,5 & \\
\hline Tarragona & 2,2 & & 1,9 & \\
\hline Resto & 18,0 & & 13,3 & \\
\hline Lengua habitual & & & & \\
\hline Catalán & 48,8 & & 50,5 & \\
\hline Castellano & 28,0 & & 33,7 & \\
\hline Indiferente & 23,2 & & 15,9 & \\
\hline Situación laboral antes del acceso & & & & \\
\hline En paro & 17,4 & & 36,2 & \\
\hline Estudiante & 2,2 & & 2,3 & \\
\hline Trabajo asalariado estable & 66,1 & & 50,5 & \\
\hline Trabajo asalariado esporádico & 5,6 & & 3,9 & \\
\hline Otros & 8,7 & & 7,2 & \\
\hline & & & \\
\hline & & & \\
\hline
\end{tabular}


De los datos recogidos en la tabla 2 hay que destacar lo siguiente: en primer lugar podemos apreciar que la feminización del colectivo es realmente notable si únicamente nos atenemos a los dos procesos selectivos sometidos a análisis, aumentando la proporción de aspirantes femeninas en 9 puntos en un solo año. También es destacable que la edad media del colectivo (34 años) se sitúa por encima de lo que es habitual en el acceso a otros grupos funcionariales de similares características, como por ejemplo el cuerpo de Mossos d'Esquadra (27,4 años en la convocatoria de 2008). Esta realidad puede tener su explicación en el escaso atractivo y prestigio que la profesión penitenciaria tiene entre la población en general (Bensimon, 2004; Cuaresma y Nicolás, 2006, 2008; Garriga, 2000; Ramió, 2006), lo que provoca que ésta no sea una primera opción laboral para los aspirantes. También puede relacionarse con el hecho que los procesos de oposición a este cuerpo sean muy escasamente difundidos por la administración convocante, lo que a su vez explica que el $48 \%$ de los aspirantes provengan del entorno familiar y de amistad de los funcionarios ya en ejercicio (Cuaresma y Nicolás, 2008).

Por último es destacable que en los datos relativos a la situación laboral de los aspirantes pueden ya apreciarse los efectos de la crisis económica que en 2008 era ya evidente, al aumentar en un $19 \%$ el número de aspirantes sin empleo respecto al año anterior.

Los cuestionarios de recogida de datos cumplimentados por ambos grupos fueron $\operatorname{dos}^{7}$ :

- Cuestionario número 1 (Pre): cumplimentado en la primera jornada del curso de formación inicial selectiva.

- Cuestionario número 2 (Post): al finalizar la formación teórica correspondiente al curso de formación inicial.

La tasa de respuesta en ambas convocatorias fue del $100 \%$ en el primer cuestionario, y solo siete individuos no contestaron al segundo en la convocatoria

\footnotetext{
${ }^{7}$ El grupo de aspirantes correspondientes a la convocatoria de 2007 cumplimentó un cuestionario más, una vez pasados seis meses desde su incorporación al puesto de trabajo como funcionario de carrera. No obstante, pese a considerar esta información enormemente reveladora del efecto positivo de la formación inicial, no se ha incluido en este trabajo atendiendo a la necesaria comparación de los datos entre ambos grupos. El lector interesado puede consultar los resultados correspondientes al estudio completo (Cuaresma y Nicolás, 2008) en la página web del CEJFE: www.gencat.cat/justicia/cejfe, tal y como se indica en la bibliografía.
} 
correspondiente a 2007, en 2008 también dejó de contestar un número similar. Estos resultados han de ser valorados como muy satisfactorios, más aún si tenemos en cuenta la resistencia que tradicionalmente ofrecen los trabajadores penitenciarios a participar en este tipo de actividades, a facilitar sus datos personales y a manifestar abiertamente su pertenencia a este colectivo a causa de los riesgos vinculados a la actividad profesional que desempeñan y a la percepción de rechazo por parte de la población en general (Crawley, 2004; Cuaresma y Nicolás, 2006; De Diego et al., 1997; Durán, 2007).

En total se valoraron 102 variables. Todas ellas, excepto las relativas a los datos socio-demográficos que encabezaban los cuestionarios, fueron reordenadas de forma aleatoria, permitiendo este método de respuesta expresar el grado de acuerdo o desacuerdo mediante una escala tipo Likert de cinco opciones (Totalmente en desacuerdo, En desacuerdo, Indiferente, De acuerdo, Totalmente de acuerdo) con una valoración de 1 a 5, que a los efectos de análisis fueron simplificadas a tres.

En cada una de las variables los aspirantes debían escoger la respuesta que considerasen más adecuada. No había por tanto respuestas correctas ni incorrectas, cada opción de respuesta permitía ubicar a los aspirantes actitudinalmente en función del grado de acuerdo con las cuestiones planteadas.

La información recopilada fue incorporada a una base de datos realizada al efecto y analizada posteriormente mediante el programa de análisis estadístico Statistical Package for the Social Sciences 18 (SPSS 18.0).

\subsection{Variables analizadas}

Con la finalidad de valorar los cambios actitudinales experimentados por los individuos integrantes de la muestra se establecieron siete escalas de valoración que, tal y como ya se ha indicado, guardan una estrecha relación con las competencias y cometidos profesionales de un funcionario penitenciario. Estas escalas, que serán brevemente descritas a continuación, son las enunciadas como variables en la tabla 3 junto a sus estadísticos descriptivos.

Las escalas fueron seleccionadas tomando en consideración (a) un criterio semántico y (b) de análisis de contenido, seleccionando aquellas que mayor relación 
tuvieran con el perfil de competencias definido para el colectivo y con el diseño docente del curso selectivo.

Tabla 3: Estadísticos descriptivos para las variables

\begin{tabular}{|c|c|c|c|c|c|c|}
\hline & & $\bar{X}$ & Mín & Máx & $\mathbf{M}_{\text {ed }}$ & SD \\
\hline $\begin{array}{l}\text { I. Actitudes hacia el trabajo } \\
\text { de funcionario } \\
\text { penitenciario }\end{array}$ & $\begin{array}{l}12 \text { ítems, } \\
\alpha=, 64(, 79)\end{array}$ & 17,27 & 1 & 19 & 18 & 2,50 \\
\hline II. Actitudes hacia los internos & $\begin{array}{l}34 \text { ítems, } \\
\alpha=, 84(, 84)\end{array}$ & 45,41 & 9 & 55 & 48 & 5,92 \\
\hline III. Apoyo a la rehabilitación & $\begin{array}{l}9 \text { ítems, } \\
\alpha=, 56(, 86)\end{array}$ & 13,89 & 0 & 15 & 15 & 1,90 \\
\hline $\begin{array}{l}\text { IV. Actitudes hacia la } \\
\text { disuasión }\end{array}$ & $\begin{array}{l}5 \text { ítems, } \\
\alpha=, 44(, 80)\end{array}$ & 7,53 & 2 & 8 & 8 & 1,06 \\
\hline $\begin{array}{l}\text { v. Orientación profesional al } \\
\text { servicio a las personas }\end{array}$ & $\begin{array}{l}8 \text { ítems, } \\
\alpha=, 63(, 70)\end{array}$ & 9,13 & 1 & 10 & 10 & 1,58 \\
\hline $\begin{array}{l}\text { VI. Motivación laboral } \\
\text { intrínseca }\end{array}$ & $\begin{array}{l}6 \text { ítems, } \\
\alpha=, 37(, 82)\end{array}$ & 5,85 & 0 & 6 & 6 & ,48 \\
\hline $\begin{array}{l}\text { VII. Auto-eficacia en el trabajo } \\
\text { penitenciario }\end{array}$ & $\begin{array}{l}15 \text { ítems, } \\
\alpha=, 59(, 82)\end{array}$ & 21,46 & 8 & 22 & 22 & 1,40 \\
\hline
\end{tabular}

$\alpha=$ Coeficiente Alfa de Cronbach de consistencia interna. Entre paréntesis se indican los mejores índices hallados en otras investigaciones.

Se analizó la distribución de los valores mediante la prueba de KolmogorovSmirnov y en todos los valores se obtuvieron estadísticos $Z$ significativos, por lo que las distribuciones de los valores analizados se aproximan adecuadamente a la distribución normal.

\section{Escala I - Actitudes hacia el trabajo como funcionario penitenciario:}

Fue desarrollada originalmente por Robinson et al. (1993). Permite evaluar el interés general de un individuo en el ámbito penitenciario, más concretamente en las áreas relativas a la preferencia por la carrera profesional en el medio carcelario y el contacto con los internos, la opinión sobre la visión que la sociedad tiene del trabajo desarrollado y el desafío profesional que éste pueda representar. Las puntuaciones altas en esta escala indican interés en este trabajo y se valoran positivamente. 


\section{Escala II - Actitudes hacia los internos:}

El original de esa escala fue desarrollado por Melvin et al. (1985). Las puntuaciones altas indican que la visión que se tiene de los internos es la de personas normales capaces de llevar a cabo cambios positivos, en sentido contrario las puntuaciones bajas reflejan una visión de ellos como personas desviadas y escasamente susceptibles al cambio.

\section{Escala III - Apoyo a la rehabilitación:}

Esta escala de nueve ítems fue creada por Cullen et al. (1989) con la intención de medir la orientación individual hacia la rehabilitación de los delincuentes. La rehabilitación, y los programas de tratamiento en particular, tienen un más que notable efecto positivo evidenciado por numerosas evidencias empíricas. Así por ejemplo, en el caso particular de los delincuentes sexuales Lösel (2002) informa de una medida del efecto global de $r=.12$, resultados coincidentes con los obtenidos por Redondo et al. (2005) en un estudio realizado en la prisión barcelonesa de Brians 1, quienes concluyeron que la efectividad del tratamiento específico para delincuentes sexuales allí aplicado reducía en un $14,1 \%$ la tasa de reincidencia. Por otra parte la literatura científica ha asentado sobradamente la importancia del apoyo y la implicación de los terapeutas que trabajan con delincuentes en la consecución de resultados positivos (Redondo, 2008; Tellier y Serin, 2001; Ward y Brown, 2004), y ello se asume como igualmente válido para los profesionales responsables de la seguridad, o también llamados de primera línea, en un sistema penitenciario en el que, como en el catalán, la relación de estos con los internos es intensiva y continua.

\section{Escala IV - Actitudes hacia la disuasión:}

La escala de disuasión fue adaptada de la sub-escala elaborada por Cullen et al. (1989). El propósito de esta escala es evaluar las actitudes de los funcionarios penitenciarios respecto a la prisión como medio adecuado para disuadir a los 
delincuentes de cometer nuevos delitos. Las más altas puntuaciones indican una mayor tendencia al uso de la prisión como castigo y respuesta adecuada ante las transgresiones legales.

\section{Escala V - Orientación profesional al servicio a las personas:}

Desarrollada por Robinson et al. (1993) esta escala mide el nivel de orientación hacia el servicio a las personas que evidencian los funcionarios de prisiones.

\section{Escala VI: Motivación laboral intrínseca}

Lo sometido a análisis en esta escala es la motivación intrínseca para el ejercicio de la profesión. Los resultados nos permiten conocer qué satisface a un funcionario penitenciario en su ejercicio profesional.

La motivación laboral fue analizada utilizando como base la escala Warr-CookWall (1979) (Bensimon, 2004 y 2005a). Se trata de un instrumento estándar para medir la motivación laboral de los aspirantes y nuevos trabajadores, que ha sido ampliamente utilizado en el ámbito de las profesiones sanitarias. Las mayores puntuaciones de la escala indican una más alta motivación laboral.

\section{Escala VII - Auto-eficacia en el trabajo penitenciario:}

Medir la eficacia profesional en un entorno como el penitenciario es una tarea difícil, y en esta ocasión aún más ya que la valoración de auto-eficacia debían hacerla la mayor parte de los aspirantes de forma evocativa. Como en cualquier profesión prestacional destinada a las personas, los logros y los objetivos profesionales son difícilmente cuantificables, pero en una prisión las demandas y exigencias derivadas de las muchas carencias personales, sociales, relacionales, formativas y de cualquier otra índole que presenta buena parte de la población reclusa hacen que "ningún otro miembro del personal reciba tantas peticiones, quejas y agravios por parte de los internos como los funcionarios de prisiones" (Bensimon, 2004; p. 20), aquellos a los 
que Crawley (2004) y también Tellier y Serin (2001) denominan funcionarios de primera línea. Esta escala fue desarrollada por Bensimon (2004).

\subsection{Técnicas de análisis}

Para llevar a cabo el estudio de cada una de las escalas actitudinales, en relación con el género de los aspirantes y con el posible efecto atribuible a la formación, se procede a aplicar el procedimiento estadístico de análisis de la varianza unidireccional (ANOVA one way) que permite detectar diferencias significativas entre los diversos grupos (hombres Vs. mujeres / pre-formación Vs. post-formación). Así para cada uno de los grupos se ejecuta un análisis de la varianza sobre cada una de las siete escalas, tomando como variables independientes la formación recibida y el género de los aspirantes. Este tipo de análisis se considera el más adecuado atendiendo a las características de las variables, independencia y distribución normal de los valores.

Mediante este procedimiento de análisis se logra dividir la variabilidad observada en componentes independientes atribuibles por un lado al efecto de la formación recibida y por el otro al género de los aspirantes, es decir, los valores $F$ significativos indican una relación entre la variable dependiente (actitudes profesionales) y las variables independientes (formación y género) de este estudio.

De forma complementaria también se analiza el tamaño del efecto (Cohen, 1992), estadístico que permite valorar la relevancia o magnitud de las diferencias encontradas mediante el procedimiento ANOVA. Este estadístico se corresponde con la diferencia tipificada, esto es, la diferencia entre dos medias dividida por una desviación típica. Su fórmula básica es la siguiente:

$$
d=\frac{\bar{X}_{1}-\bar{X}_{2}}{\sigma}
$$




\section{Resultados}

En cuanto a la primera de las hipótesis planteadas, en el momento de iniciar el curso selectivo, al contestar los aspirantes al cuestionario número 1 (Pre), la mayor parte de las escalas evidenciaron resultados significativos en el contraste inter-géneros (tabla 4).

Tabla 4: ANOVA y tamaño del efecto inter-géneros, antes y después de la formación

\begin{tabular}{|ll|c|c|}
\hline \multicolumn{1}{|c|}{ Escalas } & Pre & Post \\
\hline I. & $\begin{array}{l}\text { Actitudes hacia el trabajo de } \\
\text { funcionario penitenciario }\end{array}$ & $F(1,617)=9.958$, & $F(1,595)=7.272$, \\
& & $p<.01$ & $p<.01$ \\
$(d=.25)$ & $(d=.18)$ \\
\hline II. & Actitudes hacia los internos & $F(1,609)=6.201$, & $F(1,592)=.454$, \\
& & $p<.05$ & $p<.501$ \\
& $(d=.24)$ & $(d=.04)$ \\
\hline III. & Apoyo a la rehabilitación & $F(1,616)=.694$, & $F(1,599)=1.430$, \\
& & $p<.405$ & $p<.232$ \\
& & $(d=.08)$ & $(d=.11)$ \\
\hline IV. & Actitudes hacia la disuasión & $F(1,620)=2.638$, & $F(1,605)=2.499$, \\
& & $p<.105$ & $p<.114$ \\
& $(d=.18)$ & $(d=.14)$ \\
\hline V. & Orientación profesional al servicio a & $F(1,619)=7.074$, & $F(1,604)=2.980$, \\
& las personas & $p<.01$ & $p<.085$ \\
& & $(d=.22)$ & $(d=.11)$ \\
\hline VI. & Motivación laboral intrínseca & $F(1,617)=4.657$, & $F(1,602)=.438$, \\
& & $p<.05$ & $p<.509$ \\
& & $(d=.17)$ & $(d=.04)$ \\
\hline vII. Auto-eficacia en el trabajo & $F(1,618)=6.613$, & $F(1,603)=2.411$, \\
& penitenciario & $p<.01$ & $p<.121$ \\
& & $(d=.20)$ & $(d=.13)$ \\
\hline
\end{tabular}

$d=$ tamaño del efecto. Cohen (1992) sugiere que tamaños del efecto de .10 han de ser considerados pequeños, de .25 de valor medio y por encima de .40 de valor grande.

Es de destacar especialmente la escala I, Actitudes hacia el trabajo de funcionario penitenciario, siendo el tamaño del efecto de valor medio (d=.25); Actitudes hacia los internos, con un tamaño del efecto muy cercano al límite medio $(d=.24)$; Orientación profesional al servicio a las personas, y un tamaño del efecto de $d=.22$; 
Motivación laboral intrínseca, con tamaño del efecto $d=.17$ y Auto-eficacia en el trabajo penitenciario y tamaño del efecto $d=.20$. Por su parte las escalas Apoyo a la rehabilitación y Actitudes hacia la disuasión no resultaron ser significativas.

La diferencia de medias (tabla 5) en el momento previo a la formación entre hombres y mujeres sitúa a éstas por encima en las cinco escalas referidas.

Tabla 5: Media y desviación estándar para hombres y mujeres, antes y después de la formación.

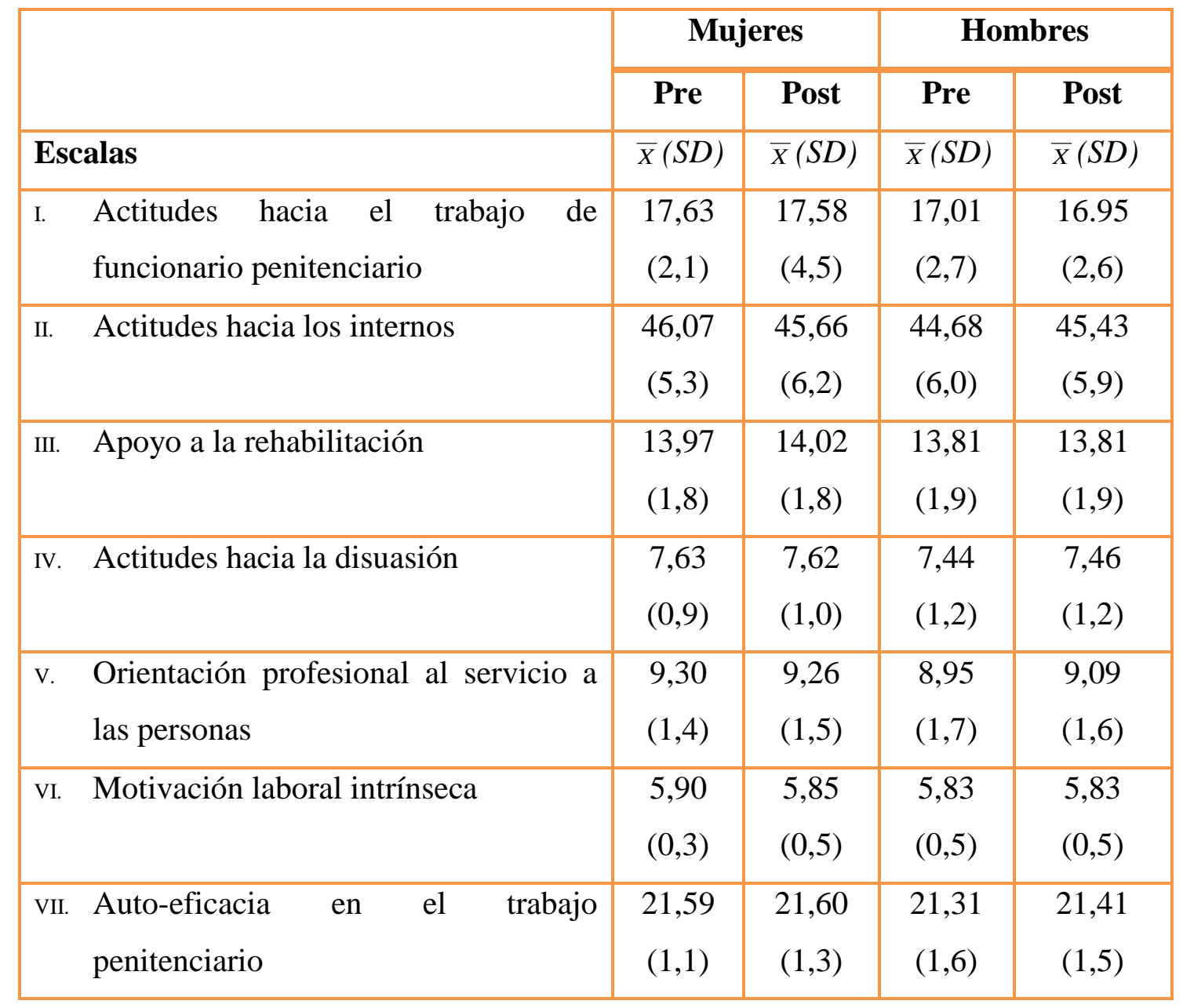

En cuanto a la segunda de las hipótesis, en el momento posterior a la formación (Post) únicamente la escala relativa a las Actitudes hacia el trabajo de funcionario penitenciario conservaba valores significativos por lo que respecta al contraste entre ambos géneros y un tamaño del efecto de $d=.18$. 
Por lo que respecta a la variación atribuible al efecto de la formación en relación con el género cabe señalar que, en la fase post únicamente se obtuvieron resultados significativos en la escala I, relativa a las Actitudes hacia el trabajo de funcionario penitenciario. Esta diferencia, en términos de tamaño del efecto, es de tamaño pequeño y de signo negativo, ya que las puntuaciones medias son inferiores tras recibir formación de lo que habían sido antes de iniciarla.

Es de destacar la mejora en las puntuaciones obtenidas por los aspirantes de género masculino en las escalas II (Actitudes hacia los internos) y V (Orientación profesional al servicio a las personas), mientras que para las mujeres las puntuaciones experimentan un acusado descenso en la escala II y algo menos en la V, los hombres aumentan sus puntuaciones medias y lo hacen de forma especialmente acentuada en la escala II, ya que si la diferencia entre géneros antes de la formación se aproximaba a un valor medio $(d=.24)$, al finalizar ésta no sólo había perdido significación sino que había desaparecido su potencia. En la escala V se observa una evolución similar. Una inicial diferencia significativa a favor de las mujeres cercana a valores medios de tamaño del efecto $(d=.22)$, que evoluciona hacia la pérdida de significación pero con un aumento en las puntuaciones obtenidas por los hombres hasta niveles cercanos a los evidenciados por sus compañeras.

En cuanto a la escala VI (Motivación laboral intrínseca), los valores de análisis indican que entre hombres y mujeres se produce una diferencia significativa de tamaño pequeño en el momento previo a la formación, desapareciendo tanto la significación como el efecto cuantificado al pasar por el proceso formativo. Los hombres se mostraron inalterables en esta escala mientras que las mujeres redujeron sus puntuaciones.

En la última de las escalas analizadas (VII. Auto-eficacia en el trabajo penitenciario) mientras las mujeres no sufrieron cambio alguno, los hombres experimentaron un reseñable aumento en su eficacia profesional percibida. La diferencia inicial entre ambos géneros $(d=.20)$ pierde no obstante significación al finalizar el curso selectivo. 


\section{Conclusiones}

\subsection{Discusión}

Se observa lo que podría denominarse un claro dimorfismo o dualidad actitudinal atribuible al género. Las mujeres parten inicialmente con mejores puntuaciones en todas las escalas, manteniendo los niveles iniciales; no obstante son los hombres los que mejoran por efecto de la formación sus peores resultados iniciales, mostrándose las mujeres refractarias a los efectos de la formación recibida, cuando no obteniendo resultados contrarios a los deseados al descender las valoraciones medias.

Se puede concluir que, en lo tocante al efecto de la formación, esta ejerció un influjo igualador entre los aspirantes de ambos géneros, homogeneizando sus actitudes profesionales, pero que el efecto es debido fundamentalmente a la mejora actitudinal que indujo en los hombres.

Estos resultados son consecuentes con los obtenidos por Cook y Lane (2012), evidenciando consistencia en la discrepante valoración de hombres y mujeres respecto a los internos con los que deben trabajar a diario. Por su parte Bensimon (2005b) encontró diferencias significativas entre hombres y mujeres, tras tres meses de formación y un año de ejercicio laboral como agente penitenciario, en las escalas Apoyo a la rehabilitación, Actitudes hacia el trabajo como funcionario penitenciario y Empatía, en las que las mujeres puntuaron más alto.

En un sentido general puede afirmarse que, si bien ha resultado acreditado que las aspirantes de género femenino se aproximan al medio profesional de seguridad penitenciaria con mejores actitudes que sus compañeros varones, destacando en todas las variables analizadas, son los hombres los que se muestran más susceptibles al cambio promovido por el curso selectivo, superándolas en tres de las cinco escalas en las que la inicial diferencia apreciada entre ambos géneros resultó ser significativamente relevante. No obstante, salvo en las actitudes hacia el trabajo penitenciario (escala I), no se obtuvieron resultados significativos en las diferencias entre géneros en las medias post-formación.

En particular es reseñable el cambio experimentado en el caso de la escala relativa a las actitudes hacia los internos (escala II), ya que mientras el curso selectivo suscitó el descenso acusado de las actitudes de las mujeres, en doble proporción aumentaron las de los hombres. En esta variable, una de las más importantes del estudio 
y que más tiene que preocupar a los gestores penitenciarios, aunque se ha observado que hay una discrepancia de partida entre ambos géneros, la formación propicia una muy notable mejora. Así, si los hombres no reciben formación, manifiestan peores actitudes que las mujeres, pero esta diferencia se anula tras el curso de capacitación. El efecto corrector de la formación es muy importante en este caso, igualando las actitudes con independencia del género.

Siendo el cometido principal de estos funcionarios el de garantizar la seguridad del establecimiento y de cuantos en él comparten espacio y tiempo, su orientación al servicio a las personas resulta igualmente importante, por cuanto su rol profesional incorpora un innegable contenido asistencial. En esta variable se repite lo que ya hemos visto en la valoración general: las mujeres destacan de principio a fin, pero los hombres mejoran con la formación y prácticamente igualan a sus compañeras.

En la motivación laboral no se apreció efecto del género en relación con la formación recibida, no obstante sí es reseñable que las mujeres mostraron una más acentuada motivación que los hombres.

Finalmente en cuanto a la auto-eficacia como trabajador penitenciario, las mujeres se situaron por delante de los hombres en variación favorable obtenida a través de la formación. Los dos grupos mejoraron su percepción de eficacia profesional, y en consecuencia su seguridad en el momento de enfrentarse al trabajo que les esperaba, pero los hombres lo hicieron ligeramente más que las mujeres.

Así pues, en cuanto a las hipótesis planteadas, estos resultados permiten afirmar no solo la inicial diferencia entre géneros, sino que la formación previa y específica, orientada a la capacitación del personal penitenciario, es un elemento favorable y extraordinariamente necesario para el sistema. La formación redunda en la humanización y dignidad del sistema, y además logra unificar las actitudes de los funcionarios reduciendo el efecto del género en su ejercicio profesional. Con la formación recibida se logra limar las diferencias apreciadas que de forma natural, en su ausencia, se producirían.

\subsection{Limitaciones}


El presente trabajo se ha visto limitado por la dificultad de poder llevar a cabo un seguimiento de la evolución actitudinal de los funcionarios una vez iniciado el ejercicio efectivo de su desempeño profesional.

Este seguimiento sí se realizó no obstante en el grupo de aspirantes correspondientes a la convocatoria de 2007, a quienes se ofreció un tercer cuestionario una vez completados los seis primeros meses de ejercicio laboral (ver nota 7). Este tercer cuestionario tuvo una tasa de respuesta de únicamente el $38,7 \%$, pese a que aun habiéndose remitido por correo postal a los domicilios de los aspirantes se habilitó un procedimiento que garantizaba el anonimato de sus respuestas. Los resultados de esta tercera encuesta no resultaron estadísticamente significativos pero sí permitieron constatar "la lucha eterna que existe entre la teoría y la ejecución en cada profesión" (Bensimon, 2004; p. II), observándose una disminución del efecto atribuible a la formación y notables cambios actitudinales en ambos géneros (Cuaresma y Nicolás, 2008). Futuras investigaciones deberían intentar constatar las diferencias actitudinales entre géneros en el ejercicio profesional de la seguridad penitenciaria y tratar de corroborar el efecto beneficioso atribuible a la formación inicial selectiva una vez iniciado el ejercicio profesional efectivo.

\subsection{Implicaciones}

Las administraciones penitenciarias deberían tener en cuenta estos resultados para implementar sistemas formativos adecuados a sus necesidades, fomentando en mayor medida la valoración que los funcionarios de género masculino tengan hacia los internos, o incluso su empatía hacia ellos, tal y como apuntaban Ireland y Quinn (2007), así como realzando la valoración de su propio ejercicio profesional y mejorando la que expresan acerca de la rehabilitación como misión del sistema penitenciario.

Por otra parte, respecto de las funcionarias, convendría valorar muy especialmente su mayor resistencia a los contenidos de la formación ya que, como se ha podido apreciar, la influencia de esta en sus actitudes es bastante menor que en el caso de los hombres. 


\section{Agradecimientos}

Los autores desean agradecer al Dr. Santiago Redondo sus comentarios a la primera versión de este texto. También desean agradecer al editor de la revista y a los revisores anónimos las observaciones realizadas que, sin duda alguna, han mejorado este artículo. Parte de esta investigación se benefició, en el año 2007, de una ayuda a la investigación del Centre d'Estudis Jurídics i Formació Especialitzada, entidad autónoma del Departament de Justícia de la Generalitat de Catalunya.

\section{Referencias}

Ballbé, M. (1983). Orden público y militarismo en la España constitucional: 18121983. Madrid: Alianza Universidad.

Bensimon, P. (2004). Correctional Officer Recruits and the Prison Environment: A Research Framework. Ottawa (Canadá): Correctional Service of Canada.

Bensimon, P. (2005a). Correctional Officer Recruits During the College Training Period: An Examination. Ottawa (Canadá): Correctional Service of Canada.

Bensimon, P. (2005b). Correctional Officers and Their First Year: An Empirical Investigation. Ottawa (Canadá): Correctional Service of Canada.

Cohen, J. (1992). A Power Primer. Psychological Bulletin, 112 (1), 155-159.

Cook, C.L. y Lane, J. (2012). Examining Differences in Attitudes about Sexual Victimization among a Sample of Jail Officers: The importance of Officer Gender and Perceived Inmate Characteristics. Criminal Justice Review, 37 (2), 191-213.

Crawley, E. (2004). Doing Prison Work: The public and private lives of prison officers. Londres: Willan Publishing.

Crouch, B., y Alpert, G. (1982). Sex and occupational socialization among prison guards. Criminal Justice and Behavior, 9, 156-176.

Cuaresma, D. y Nicolás, L. (2006). La formació del funcionari penitenciari. Estudi de les necessitats formatives dels funcionaris de vigilància $i$ seguretat del sistema penitenciari català. Barcelona: Centre d'Estudis Jurídics i Formació Especialitzada.

Cuaresma, D. y Nicolás, L. (2008). Actituds professionals dels funcionaris penitenciaris. L'efecte de la formació inicial. Barcelona: Centre d'Estudis Jurídics i Formació Especialitzada. Disponible en: http://www.gencat.cat/justicia/cejfe 
Cullen, F.T., Lutze, F.E., Link, BG. y Wolfe, N.T. (1989). Correctional Orientation of Prison Guards: Do Officers Support Rehabilitation? Federal Probation Quarterly, 53(1), 33-42.

De Diego, N., Durán, M. A., Rius, M. J. y Enríquez, F. J. (1997). El stress asistencial (Burnout) en los funcionarios de prisiones. Boletín criminológico, 26.

Durán, A. (2007). Estrés laboral y burnout en funcionarios de prisiones. En Cerezo, A.I. y Benítez, M.J. (Coord.). La prisión en España: una perspectiva criminológica. Granada: Comares.

Farkas, M.A. (1999). Correctional officer attitudes toward inmates and working with inmates in a 'get tough' era. Journal of Criminal Justice, 27(6), 495-506.

Farnworth, L. (1992). Women Doing a Man's Job: Female Prison Officers Working in a Male Prison. Australian \& New Zealand Journal of Criminology, 25, 278-296.

Garriga, G. (2000). La presó Model entre 1962 i 1983. En Solé, J.M. [et al.]. Història de la presó Model de Barcelona. Barcelona: Pagès editors.

Giménez-Salinas, E. (1990). Actualización profesional del funcionario de prisiones. Eguzquilore, 4, 51-62.

Goffman, E. (1970). Estigma, la identidad deteriorada. Buenos Aires: Amorrortu.

Gordon, J., Proulx, B. y Grant, P. (2012). Trepidation among the "keepers": Gendered Perceptions of Fear and Risk of Victimization among Corrections Officers. American Journal of Criminal Justice, 37(3), 1-21.

Griffin, M. L. (2012). From resistance to integration: The influence of gender in the corrections work environment. En C. M. Renzetti, S. L. Miller y A. R. Gover (Eds.). Routledge International Handbook of Crime and Gender Studies, 279.

Ireland, J. y Quinn, K. (2007). Officer Attitudes Towards Adult Male Prisoners Who Self-Harm: Development of an Attitudinal Measure and Investigation of Sex Differences. Aggresive Behavior, 33, 63-72.

Jar, G. (1995). Modelo policial español y policías autónomas. Madrid: Dykinson.

Kauffman, K. (1988). Prison officers and their World. Cambridge: Harvard University Press.

Lai, Y.L., Wang, H.M. y Kellar, M. (2012). Workplace violence in correctional institutions in Taiwan: A study of correctional officers' perceptions. International Journal of Comparative and Applied Criminal Justice, 36 (1), 1-23.

Lambert, E., Hogan, N., Altheimer, I. y Wareham, J. (2010). The Effects of Different Aspects of Supervision Among Female and Male Correctional Staff: A Preliminary Study. Criminal Justice Review, 35 (4), 492-513. 
Lavoie, J., Connolly, D. y Roesch, R. (2006). Correctional Officers' Perceptions of Inmates with Mental Illness: The Role of Training and Burnout Syndrome. International Journal of Forensic Mental Health, 5 (2), 151-166.

Liebling, A y Price, D. (2001). The prison officer. Leyhill: HM Prison Service.

López, D. (1987). El aparato policial en España. Barcelona: Ariel.

Lösel, F. (2002). "¿Sirve el tratamiento para reducir la reincidencia de los delincuentes sexuales?". En Redondo, S. (Coord.) Delincuencia sexual y sociedad. Barcelona: Ariel.

Luque, M.E., Redondo y S, Funes, J. (1992). Avaluació de la formació inicial en una mostra de funcionaris penitenciaris. Barcelona: Centre d'Estudis Jurídics i Formació Especialitzada.

Malochet, G. (2004). À l'école de la détention: quelques aspects de la socialisation professionnelle des surveillants de prison. Sociologie du travail, 46(2), 168-186.

Malochet, G. (2005). Dans l'ombre des hommes: La féminisation du personnel de surveillance des prisons pour hommes. Sociétés Contemporaines, 59, 199-220.

Marín, M. (1990). La profesión de policía. Madrid: Centro de investigaciones sociológicas.

Melvin, K., Grambling, L. y Gardner, W. (1985). A Scale to Measure Attitudes toward Prisoners. Criminal Justice and Behavior, 12 (2), 241-253.

Morgan, R., Van Haveren, R. y Pearson, C. (2002). Correctional Officer Burnout: Further Analyses. Criminal Justice and Behavior, 29 (2), 144-160.

Motiuk, L. L. y Serin, R. C. (Eds.). (2001). Compendium 2000 on effective correctional programming. Ottawa: Correctional Service Canada.

Newbold, G. (2005). Women officers working in men's prisons. Social Policy Journal of New Zealand, 25, 105-117.

Paoline, E., Lambert, E. G. y Hogan, N. L. (2006). A Calm and Happy Keeper of the Keys. The Impact of ACA Views, Relations With Cowerkers, and Policy Views on the Job Stress and Job Satisfaction of Correctional Staff. The Prison Journal, $86(2), 182-205$.

Pinker, S. (2011). The Better Angels of Our Nature. New York: Viking.

Ramió, C. (2006). Repensar la presó: Alternatives organitzatives a una institució tradicionalment jeràrquica. Ponencia presentada en Congreso penitenciario internacional: La función social de la política penitenciaria. Barcelona. Disponible

en: http://www20.gencat.cat/docs/Justicia/Documents/ARXIUS/doc_18876834_1.pdf 
Redondo, S. (2008). Manual para el tratamiento psicológico de los delincuentes. Madrid: Ediciones Pirámide.

Redondo, S., Navarro, J.C., Martínez, M., Luque, E. y Andrés, A. (2005). Evaluación del tratamiento psicológico de los agresores sexuales en la prisión de Brians. Boletín criminológico, 79 .

Robinson, D., Porporino, F. J. y Simourd, L. (1993). The influence of career orientation on support for rehabilitation among correctional staff. The Prison Journal, 73, 162-177.

Sarasa, S. (1990). Actitudes y expectativas de los aspirantes a funcionario penitenciario. Informe de la primera fase del trabajo de investigación: La situación profesional y las expectativas de los funcionarios penitenciarios. Barcelona: Centre d'Estudis Jurídics i Formació Especialitzada.

Taxman, F. S. y Gordon, J. A. (2009). Do fairness and equity matter? An examination of organizational justice among correctional officers in adult prisons. Criminal Justice and Behavior, 36, 695-711.

Tellier, C. y Serin, R. C. (2001). The role of staff in effective program delivery. En L. L. Motiuk y R. C. Serin (Eds.), Compendium 2000 on effective correctional programming. Ottawa: Correctional Service Canada.

Torrente, D. (1997). La sociedad policial. Poder, trabajo y cultura en una organización local de policía. Madrid: Centro de investigaciones sociológicas.

Ward, T. y Brown, M. (2004). The Good Lives Model and conceptual issues in ofender rehabilitation. Psychology, Crime \& Law, 10 (3), 243-257.

Warr, P., Cook, J. y Wall, T. (1979). Scales for the measurement of some work attitudes and aspects of psychological well-being. Journal of Occupational Psychology, 52, $129-48$.

Williamson, H. E. (1990). The Corrections Profession. Newbury Park: Sage Publications.

David Cuaresma es Graduado en Criminología y Política Criminal, Licenciado en Criminología y Diplomado en Estudios Avanzados en el programa de doctorado de Personalidad y Comportamiento de la Universitat de Barcelona. En la actualidad es mando intermedio de los servicios penitenciarios de Catalunya y profesor asociado de Criminología en la Universitat de Barcelona. Realiza su tesis doctoral en el inicio y desarrollo de las carreras criminales, lo que centra sus intereses de investigación junto al estudio del comportamiento violento, los métodos de prevención delictiva, los sistemas de ejecución penal y el funcionamiento de los cuerpos policiales y penitenciarios. Es presidente de la Asociación Catalana de Criminólogos. 
Laura Nicolás es Graduada en Criminología y Política Criminal y Licenciada en Criminología por la Universitat de Barcelona. En la actualidad es profesora asociada de Criminología de esa misma universidad. Sus principales áreas de interés son la predicción, prevención y tratamiento de la conducta delictiva, atención a las víctimas, delincuencia juvenil y el inicio y desarrollo de las carreras delictivas. 